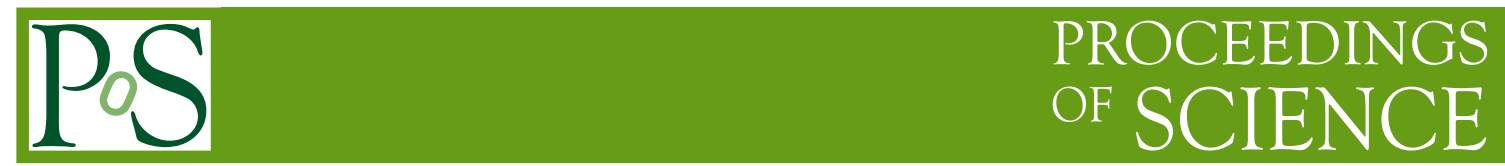

\title{
Challenges for New Physics in the Flavor Sector
}

\section{Wolfgang Altmannshofer*}

Perimeter Institute for Theoretical Physics

31 Caroline Street North, Waterloo, Ontario, Canada N2L 2 Y5

E-mail: waltmannshofereperimeterinstitute.ca

In this talk I discuss various challenges for new physics in the flavor sector: (i) the Standard Model flavor puzzle; (ii) the new physics flavor puzzle; (iii) recent anomalies in low energy flavor observables, in particular in rare $b \rightarrow$ sll decays, like the anomaly in the $B \rightarrow K^{*} \mu^{+} \mu^{-}$ angular distribution and the hint for lepton flavor universality violation in $B \rightarrow K \mu^{+} \mu^{-}$versus $B \rightarrow K e^{+} e^{-}$.

Flavor Physics \& CP Violation 2015

May 25-29, 2015

Nagoya, Japan

${ }^{*}$ Speaker. 


\section{Introduction}

Despite its impressive success, there are many good reasons to believe that the Standard Model (SM) of particle physics has to be extended by new physics (NP). Considering the SM as the low energy effective theory of a more complete model, the SM Lagrangian can be extended by higher dimensional operators that are suppressed by powers of the NP scale $\Lambda$. Concentrating on the flavorful terms one can write

$$
\mathscr{L}_{\text {flavor }}=\mathscr{L}_{\text {flavor }}^{(4)}+\frac{1}{\Lambda} \mathscr{L}_{\text {flavor }}^{(5)}+\frac{1}{\Lambda^{2}} \mathscr{L}_{\text {flavor }}^{(6)}+\cdots
$$

where $\mathscr{L}_{\text {flavor }}^{(4)}$ are the renormalizable interactions and $\mathscr{L}_{\text {flavor }}^{(5)}, \mathscr{L}_{\text {flavor }}^{(6)}$ contain operators of dimension 5 and 6, respectively. The renormalizable terms consist of the Yukawa couplings of the Higgs boson to the SM fermions that, after electro-weak symmetry breaking, lead to the masses of the SM quarks and charged leptons as well as quark flavor mixing. At the dimension 5 level a single operator exists (up to flavor assignments). It violates lepton number and after electro-weak symmetry breaking provides Majorana masses for the SM neutrinos and flavor mixing in the lepton sector. The smallness of neutrino masses points to a NP scale of $O\left(10^{14}\right) \mathrm{GeV}$ that suppresses this operator. At the dimension 6 level there exists a large set of operators that violate flavor [1]. In this talk I will concentrate on some of the challenges that are connected to $\mathscr{L}_{\text {flavor }}^{(4)}$ and $\mathscr{L}_{\text {flavor }}^{(6)}$.

Challenge 1: The SM flavor puzzle. The observed masses and mixing angles of the SM fermions show a very hierarchical pattern. While the SM Yukawa couplings allow to accommodate the observed flavor structure, no explanation of the origin of the observed hierarchies is given in the SM. In Sec. 2, I will describe a radiative fermion mass model that attempts to explain the hierarchies in terms of loop factors.

Challenge 2: The NP flavor puzzle. Most experimental results on Flavor Changing Neutral Current (FCNC) processes are in impressive agreement with SM predictions. This implies that many flavor violating dimension 6 operators (and, in turn, many NP models that introduce new sources of flavor violation) are subject to extremely stringent constraints. I will briefly expand on this in Sec. 3.

Challenge 3: Flavor anomalies. In some low energy flavor observables discrepancies between SM predictions and experimental results at the $\sim 3 \sigma$ level have emerged recently. In Sec. 4, I will briefly review some of the anomalies. I will discuss which type of NP could give an explanation of the current data and to which extend it is possible to distinguish between NP explanations and underestimated hadronic effects.

\section{The Standard Model Flavor Puzzle}

Many approaches exist that attempt to explain the hierarchical flavor structure of the SM starting from order one input parameters. Among them are Froggatt-Nielsen models [2], models with flat or warped extra dimensions [3] and models with radiatively generated fermion masses [4]. Models of radiative fermion masses explain the hierarchical pattern in the mass spectrum and mixing angles of the SM quarks and leptons in terms of loop factors. Recently, various models of that type have been discussed [5, 6]. Here I describe the framework of [6], where the masses and 
mixing angles of the SM fermions are generated through loops of their superpartners that exhibit an anarchical structure for the soft masses.

It was found in [6] that the Minimal Supersymmetric Standard Model (MSSM) with flavor anarchic squark and slepton soft masses contains all necessary ingredients to generate a fully hierarchical spectrum of quarks and leptons. At tree level, rank 1 Yukawas couple the Higgs only to the fermions of the 3rd generation. Gaugino-sfermion loops generate Yukawa couplings for the 1st and 2nd generation fermions. Yukawa couplings for both 1st and 2nd generation fermions are generated at 1-loop. Nevertheless, a novel mechanism ensures a splitting of $O(100)$, without introducing small parameters or symmetries. Indeed, the loop induced Yukawa matrices are approximately rank 1 and, in combination with the rank 1 tree level Yukawas, they give Yukawa couplings containing one very small eigenvalue. One can identify the corresponding eigenstates with the 1st generation and thus obtain a fermion mass spectrum that is fully hierarchical. Setting for simplicity all masses of supersymmetric (SUSY) particles equal and working in leading order mass insertion approximation and strictly at 1 loop, one finds for example in the down quark sector

$$
\frac{m_{s}}{m_{b}} \simeq \frac{\alpha_{s}}{4 \pi} \frac{2}{9} \frac{\left|m_{\tilde{g}} \mu\right|}{m_{\tilde{q}}^{2}} \tan \beta\left|\delta_{23}^{L} \delta_{b s}^{R}\right|, \quad \frac{m_{d}}{m_{b}} \simeq \frac{\alpha_{s}}{4 \pi} \frac{2}{225} \frac{\left|m_{\tilde{g}} \mu\right|}{m_{\tilde{q}}^{2}} \tan \beta\left|\delta_{12}^{L} \delta_{s d}^{R} \delta_{23}^{L} \delta_{b s}^{R}\right|,
$$

where the mass insertions $\delta_{i j}^{A}$ parameterize the relative size of the flavor off-diagonal squark masses squared with respect to an average squark mass squared $m_{\tilde{q}}^{2}$. Large $\tan \beta$ and $O(1)$ mass insertions lead easily to a realistic hierarchical spectrum.

Also mixing in the quark sector is typically very hierarchical. Mixing between the quarks of the 1 st and $3 \mathrm{rd}$ as well as the $2 \mathrm{nd}$ and $3 \mathrm{rd}$ generation is 1 loop suppressed. Mixing between the 1 st and 2 nd generation is small due to the approximate rank 1 nature of the loop induced Yukawa matrix.

The minimal setup leads to hierarchical fermion masses and quark mixing angles that agree well with observation at the qualitative level. At the quantitative level, most observed values of the flavor parameters can be accommodated in the large $\tan \beta$ regime of the MSSM, with the exception of the Cabibbo angle and the muon mass (see Fig. 1). A Cabibbo angle in agreement with the experimental measurement can be accommodated if flavor violating trilinear couplings are allowed in the model. The measured muon mass can be obtained by allowing for hard SUSY breaking that leads to lepton-slepton-bino couplings larger than the hypercharge gauge coupling by a factor of few. Alternatively, enlarging the SM gauge group by $U(1)_{B-L}$ can give large enough contributions to the muon mass through loops of $B-L$ gauginos.

Due to the anarchic squark and slepton masses, the framework of [6] is subject to strong constraints from low energy flavor observables. In particular, CP violation in Kaon mixing generically leads to a lower bound on the masses of squarks and gluinos of the order of $m_{\mathrm{SUSY}} \gtrsim O(1) \mathrm{PeV}$.

\section{The New Physics Flavor Puzzle}

Any NP that introduces new generic sources of flavor violation is severely constrained by the measurement of low energy flavor observables. Among the dimension 6 operators in (1.1), there are many four fermion contact interactions that contribute to meson mixing. If the coefficients of these operators are $O(1)$, the NP scale that suppresses them has to be extremely high. In particular, 

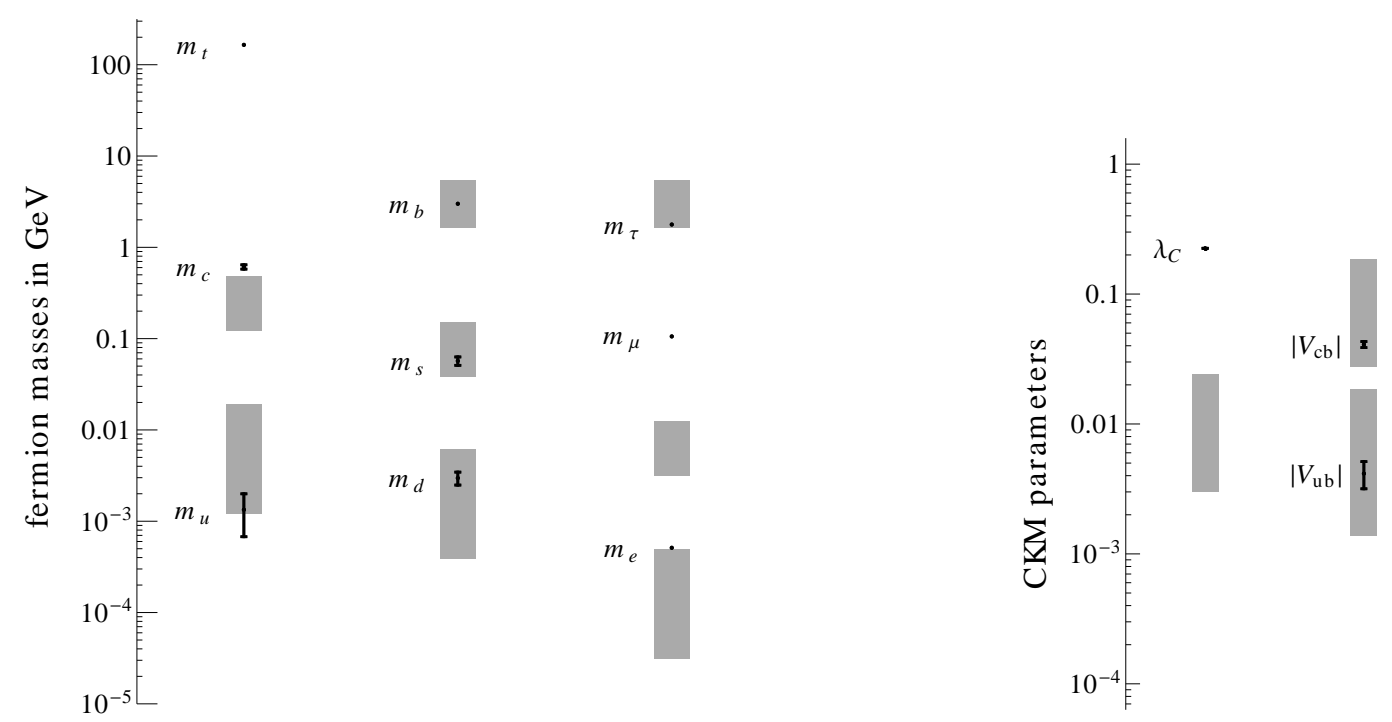

Figure 1: Generic predictions for the hierarchies in the SM fermion masses and CKM mixing elements in a minimal high scale SUSY model with radiatively induced SM fermion masses (gray boxes). Shown in black are also the observed fermion $\overline{\mathrm{MS}}$ masses at the electro-weak scale and CKM elements, with their $2 \sigma$ uncertainties. (from [6])

the scale of some operators that contribute to $\mathrm{CP}$ violation in Kaon mixing is constrained at a level of few $\times 10^{5} \mathrm{TeV}$ [7]. CP violation in D meson mixing probes scales of the order of $O\left(10^{4}\right) \mathrm{TeV}$.

While the constraints that can be put at the level of the dimension 6 operators are certainly impressive, in many NP models contributions to low energy flavor observables are suppressed at least by some amount. For example, in SUSY models with unbroken $R$ parity, NP contributions to FCNC processes are suppressed by a loop factor. Nevertheless, NP models that contain new generic sources of flavor mixing typically lead to excessive contributions to many low energy flavor observables unless the masses of the new degrees of freedom are far above the $\mathrm{TeV}$ scale. For example, in models where SUSY breaking is mediated by Planck suppressed operators it is well known that squark and slepton soft masses generically contain $O(1)$ sources of flavor violation. As shown in Fig. 2, already with current sensitivities, some low energy observables can probe squarks and sleptons with masses in the $(10-1000) \mathrm{TeV}$ range in such a case $[8,9]$. In many cases the experimental sensitivities are expected to improve significantly in the next decade and a large host of low energy observables will be able to probe flavor anarchic squarks and sleptons far above the direct reach of current particle colliders. Among the most sensitive processes are $\mathrm{CP}$ violation in Kaon and D meson mixing, electric dipole moments of the electron and hadronic systems, as well as lepton flavor violating processes like $\mu \rightarrow e \gamma, \mu \rightarrow 3 e$ and $\mu \rightarrow e$ conversion in nuclei.

If there is flavorful NP at the $\mathrm{TeV}$ scale, as suggested by most natural solutions to the hierarchy problem, the couplings of the new degrees of freedom have to have a highly non-generic flavor structure to avoid the stringent constraints from flavor observables. Therefore, if NP is discovered at the LHC, current and improved future results on flavor observables will be invaluable in identifying viable mechanisms that control this non-generic flavor structure of the NP. If no NP is discovered at LHC, flavor observables still offer the potential to indirectly uncover NP and to estimate a NP 


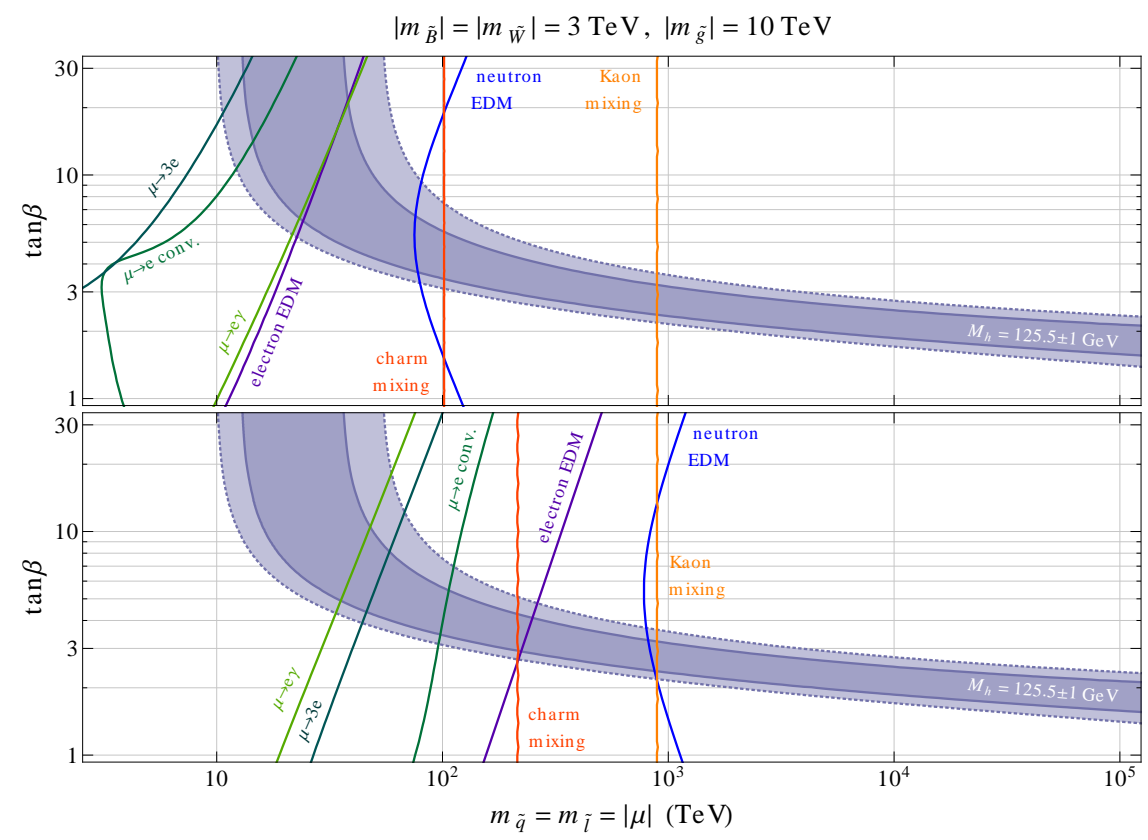

Figure 2: Sensitivity of various low energy processes in the sfermion mass vs. $\tan \beta$ plane, fixing the mass insertions to $\delta_{i j}^{A}=0.3$. The shaded band is the parameter space that in the MSSM is compatible with a Higgs mass of $m_{h}=125.5 \pm 1 \mathrm{GeV}$. The upper (lower) plot gives the reach of current (projected future) experimental results. (from [8])

scale. Given their exquisite sensitivity to mass scales beyond those accessible at the LHC, it is not unlikely that first signs of NP will appear in flavor observables.

\section{Flavor Anomalies}

In the recent years, anomalies appeared in several low energy flavor observables that can already be interpreted as hints for NP. Among them are

(i) indications for enhanced $B \rightarrow D^{(*)} \tau \nu$ rates from BaBar [10], Belle [11] and LHCb [12]. The combination of the results deviates from SM predictions [13] by more than $3 \sigma$.

(ii) the $B_{s} \rightarrow \phi \mu^{+} \mu^{-}$decay rate measured by LHCb [14], that appears suppressed by more than $3 \sigma$ with respect to the latest SM prediction $[15,16]$.

(iii) an anomaly in the $B \rightarrow K^{*} \mu^{+} \mu^{-}$angular distribution observed by LHCb [17]. The angular observable $P_{5}^{\prime}$ deviates up to $3 \sigma$ from SM predictions $[15,16,18,19,20]$.

(iv) the $\sim 2.5 \sigma$ hint for lepton flavor universality (LFU) violation in $B \rightarrow K \ell^{+} \ell^{-}$by LHCb [21].

The anomalies (ii), (iii), and (iv) are all connected to the $b \rightarrow s \mu \mu$ transition. In the following I will concentrate on those. Possible connections with the deviations in the $B \rightarrow D^{(*)} \tau \nu$ decays have been discussed in [22].

Some of the mentioned anomalies might be (at least partly) due to underestimated theory uncertainties. For example, the $B_{s} \rightarrow \phi \mu^{+} \mu^{-}$decay rate is subject to sizable uncertainties from 
form factors and CKM matrix elements. Bridging the large gap between the SM prediction and the experimental result ${ }^{1}$

$$
\begin{aligned}
& \operatorname{BR}\left(B_{s} \rightarrow \phi \mu^{+} \mu^{-}\right)_{[1,6]}^{\exp }=\left(1.29_{-0.16}^{+0.17} \pm 0.04 \pm 0.10\right) \times 10^{-7}[14], \\
& \operatorname{BR}\left(B_{s} \rightarrow \phi \mu^{+} \mu^{-}\right)_{[1,6]}^{\mathrm{SM}}=(2.4 \pm 0.3) \times 10^{-7}[15,16],
\end{aligned}
$$

would require drastic changes to the form factor normalization or to the CKM element $V_{c b}$. The observable $P_{5}^{\prime}$ that characterizes aspects of the angular distribution of the $B \rightarrow K^{*} \mu^{+} \mu^{-}$decay is independent of the overall form factor normalization and CKM elements and is therefore theoretically cleaner than the corresponding decay rate. However, $P_{5}^{\prime}$ depends on poorly understood non-factorizable hadronic effects $[23,18,20]$. The difference between the measurement and the SM prediction

$$
\left(P_{5}^{\prime}\right)_{[4,6]}^{\exp }=-0.300_{-0.156}^{+0.155} \pm 0.023[17], \quad\left(P_{5}^{\prime}\right)_{[4,6]}^{\mathrm{SM}}=-0.774 \pm 0.067[16],
$$

is much larger than estimates of such hadronic effects [24]. However, a SM explanation of the discrepancy cannot be excluded at the moment. On the other hand, the lepton flavor universality ratio $R_{K}$ is known to be a theoretically extremely clean observable. The current measurement hints towards violation of LFU

$$
R_{K}^{\exp }=\frac{\operatorname{BR}\left(B \rightarrow K \mu^{+} \mu^{-}\right)_{[1,6]}^{\exp }}{\operatorname{BR}\left(B \rightarrow K e^{+} e^{-}\right)_{[1,6]}^{\exp }}=0.745_{-0.074}^{+0.090} \pm 0.036[21], \quad R_{K}^{\mathrm{SM}} \simeq 1.00[25] .
$$

A significant deviation from the SM prediction in this observable would be a clear sign of NP.

\subsection{Global Fits to $b \rightarrow s \ell \ell$ Data}

$\mathrm{NP}$ in $b \rightarrow s \mu \mu$ transitions can be described model independently by a subset of the dimension 6 terms in the flavorful Lagrangian (1.1). It is customary to use the effective Hamiltonian valid at the scale of B mesons, where SM degrees of freedom at the electro-weak are already integrated out

$$
\mathscr{H}_{\mathrm{eff}}=\mathscr{H}_{\mathrm{eff}}^{\mathrm{SM}}-\frac{4 G_{F}}{\sqrt{2}} V_{t s}^{*} V_{t b} \frac{e^{2}}{16 \pi^{2}} \sum_{i}\left(C_{i}^{\mathrm{NP}} O_{i}+C_{i}^{\prime} O_{i}^{\prime}\right)+\text { h.c. }
$$

with the most relevant dimension six operators

$$
\begin{array}{cl}
O_{7}=\frac{m_{b}}{e}\left(\bar{s} \sigma_{\mu v} P_{R} b\right) F^{\mu v}, & O_{7}^{\prime}=\frac{m_{b}}{e}\left(\bar{s} \sigma_{\mu v} P_{L} b\right) F^{\mu v}, \\
O_{9}=\left(\bar{s} \gamma_{\alpha} P_{L} b\right)\left(\bar{\mu} \gamma^{\alpha} \mu\right), & O_{9}^{\prime}=\left(\bar{s} \gamma_{\alpha} P_{R} b\right)\left(\bar{\mu} \gamma^{\alpha} \mu\right), \\
O_{10}=\left(\bar{s} \gamma_{\alpha} P_{L} b\right)\left(\bar{\mu} \gamma^{\alpha} \gamma_{5} \mu\right), & O_{10}^{\prime}=\left(\bar{s} \gamma_{\alpha} P_{R} b\right)\left(\bar{\mu} \gamma^{\alpha} \gamma_{5} \mu\right) .
\end{array}
$$

NP in any of these operators will generically affect many processes and observables simultaneously (in particularly not only the discrepant observables but also observables that show reasonable agreement with SM predictions). Global fits of all available experimental data are therefore mandatory to identify consistent explanations of the anomalies.

\footnotetext{
${ }^{1}$ Here and in the following, subscripts like $[1,6]$ refer to ranges of di-lepton invariant mass $1 \mathrm{GeV}^{2}<q^{2}<6 \mathrm{GeV}^{2}$.
} 

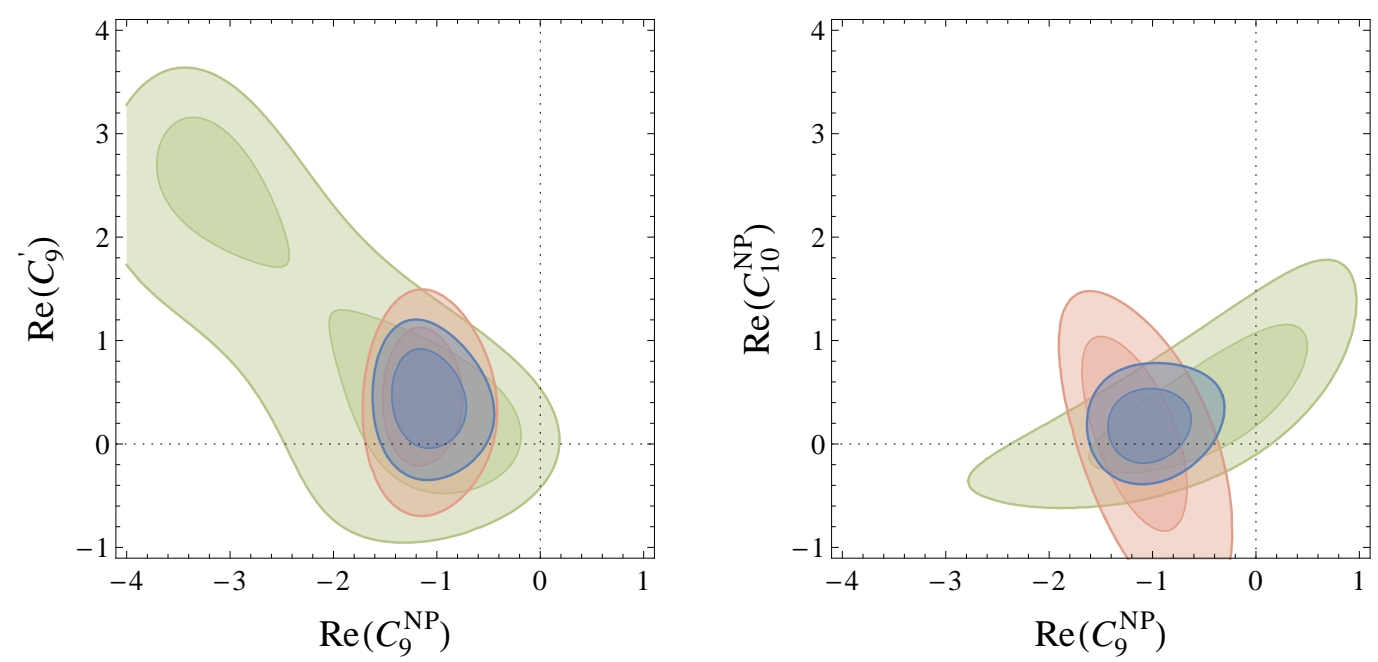

Figure 3: Favored NP parameter space in the $C_{9}-C_{9}^{\prime}$ and $C_{9}-C_{10}$ planes, taking into account all relevant data on rare decays based on the $b \rightarrow s \mu \mu$ transition. In green (red) the 1 and $2 \sigma$ region taking into account only branching ratio measurements (only angular observables). The blue contours correspond to the full fit. (from [15])

Several recent global analyses with various degrees of sophistication exist [26, 15, 19, 20, 27]. Most works find significant preference for NP in the Wilson coefficient $C_{9}$. Shown in Fig. 3 are results of the fit performed in [15] that uses state-of-the-art form factors, includes all relevant known non-factorizable corrections to the decay amplitudes and takes into account all the correlations of theoretical uncertainties between different observables and between different bins of dilepton invariant mass. Assuming that additional unknown hadronic uncertainties are estimated in a sufficiently conservative way, the $\chi^{2}$ improves significantly with a negative NP contribution to $C_{9}$. With $C_{9}^{\mathrm{NP}}=-1.07 \simeq-25 \% \times C_{9}^{\mathrm{SM}}$ it is found that

$$
\Delta \chi^{2}=\chi^{2}\left(C_{9}^{\mathrm{NP}}=-1.07\right)-\chi_{\mathrm{SM}}^{2}=13.7,
$$

where in the SM $\chi_{\mathrm{SM}}^{2} \simeq 117$ for 88 independent measurements. Concerning the hint for LFU violation, it is found that NP exclusively in the muonic decay modes leads to an excellent description of the data. There is no preference for NP in the electron modes.

The result that the best fit of the data is obtained by a NP effect in the Wilson coefficient $C_{9}$ might be worrying, as $C_{9}$ corresponds to an operator with a left-handed quark FCNC and a vectorlike coupling to muons. Unaccounted for hadronic corrections could mimic exactly such an effect. There are ways to distinguishing NP from hadronic effects. A NP explanation would be required if

- anomalies are only seen in $b \rightarrow s \mu \mu$ transitions and not in $b \rightarrow$ see (as the $R_{K}$ measurement seems to hint at), because hadronic effects are lepton flavor universal.

- anomalies are seen in $B \rightarrow K^{*} \mu^{+} \mu^{-}$angular observables like $S_{3}$ or $A_{9}$ that vanish in the absence of right-handed currents, because hadronic effects do not induce sizable right-handed currents.

- sizable values for the $B \rightarrow K^{*} \mu^{+} \mu^{-} \mathrm{CP}$ asymmetries $A_{7}, A_{8}$ or $A_{9}$ are observed, because hadronic effects do not contain large $\mathrm{CP}$ violating phases. 
On the other hand, a NP explanation would be disfavored if a good description of the anomalies is only possible with a modification of $C_{9}$ that shows a non-trivial $q^{2}$ dependence. Indeed, NP that is described by an effective Hamiltonian leads by construction to a $q^{2}$ independent modification of the Wilson coefficients. While it might be possible to construct a NP model containing light degrees of freedom that lead to a non-trivial $q^{2}$ dependence, an explanation in terms of hadronic effects would appear more plausible in such a case.

\subsection{New Physics Models for the $b \rightarrow s \ell \ell$ Anomalies}

Assuming that the anomalies in $b \rightarrow$ sll transitions are due to NP effects, one can translate the best fit values for $C_{9}$ into a NP scale $\Lambda$. For $\Delta \mathscr{H}_{\text {eff }}=O_{9} / \Lambda^{2}$, the best fit value corresponds to a scale $\Lambda \simeq 35 \mathrm{TeV}$. This is the scale of tree-level NP with $O(1)$ flavor changing $b \leftrightarrow s$ couplings and $O(1)$ couplings to muons. Smaller couplings correspond to lower scales.

Various NP models have been discussed in the literature that can explain the anomalies without violating additional model specific low and high energy constraints. Among them are lepto-quark models [28] and $Z^{\prime}$ models [29, 30]. The favored effect in $C_{9}$ can readily be accommodated in models that contain massive $Z^{\prime}$ gauge bosons with flavor changing $b \leftrightarrow s$ couplings as well as vector couplings to muons. A particularly promising candidate is a $Z^{\prime}$ boson that is associated to gauging the difference between muon and tau number $L_{\mu}-L_{\tau}$ [29]. It allows to explain the anomalies in $b \rightarrow s \mu \mu$ decays without affecting the corresponding electron modes and therefore addresses automatically the hint for lepton flavor universality violation. A $Z^{\prime}$ boson based on gauging $L_{\mu}-L_{\tau}$ is only weakly constrained by other processes. The most important probes turn out to be $B_{s}$ mixing and the process of neutrino trident production [31]. Neutrino tridents lead to a lower bound on the ratio of $Z^{\prime}$ mass and $L_{\mu}-L_{\tau}$ gauge coupling $m_{Z^{\prime}} / g^{\prime} \gtrsim 540 \mathrm{GeV}$, while $B_{s}$ mixing leads to an upper bound $m_{Z^{\prime}} / g^{\prime} \lesssim$ few TeV.

Many models that explain the anomalies make testable predictions for other processes like $B_{s} \rightarrow \mu^{+} \mu^{-}, B \rightarrow X_{s} \rightarrow \ell^{+} \ell^{-}, b \rightarrow s v \bar{v}$ decays, more lepton flavor universality ratios [15, 32, 20], and even lepton flavor violating rare $B$ decays like $B \rightarrow K \tau \mu$ [33]. If future measurements will confirm the current anomalies and if a NP origin can be clearly established, a correlated analysis of all the above processes might allow to disentangle the underlying model of NP.

\section{Summary}

Flavor remains a puzzling aspect both in the Standard Model and in models of new physics. On the one hand, there is the SM flavor puzzle: The quarks and leptons show a very hierarchical flavor structure that can be accommodated but not explained in the SM. What is the origin of the observed flavor hierarchies? On the other hand, there is the NP flavor puzzle: If there is NP at the $\mathrm{TeV}$ scale, as suggested by most natural solutions to the hierarchy problem, it has to have a highly non-generic flavor structure. What is the precise nature of the NP flavor structure? Where does it come from?

Concerning the current anomalies in flavor observables: It is likely that the discrepancies will be either diluted or sharpened with future experimental data. In the meantime it is an important task to investigate which type of NP is favored by the current anomalies, and to develop strategies to distinguish between explanations in terms of NP and possible underestimated hadronic effects. 


\section{Acknowledgements}

It is a pleasure to thank the organizers of the FPCP 2015 conference for the invitation to present this talk. I thank Stefania Gori for comments on the manuscript. Research at Perimeter Institute is supported by the Government of Canada through Industry Canada and by the Province of Ontario through the Ministry of Economic Development \& Innovation.

\section{References}

[1] B. Grzadkowski, M. Iskrzynski, M. Misiak and J. Rosiek, JHEP 1010, 085 (2010) [arXiv:1008.4884 [hep-ph]].

[2] C. D. Froggatt and H. B. Nielsen, Nucl. Phys. B 147, 277 (1979).

[3] N. Arkani-Hamed and M. Schmaltz, Phys. Rev. D 61, 033005 (2000) [hep-ph/9903417]; Y. Grossman and M. Neubert, Phys. Lett. B 474, 361 (2000) [hep-ph/9912408].

[4] S. Weinberg, Phys. Rev. Lett. 29, 388 (1972).

[5] A. Ibarra and A. Solaguren-Beascoa, Phys. Lett. B 736, 16 (2014) [arXiv:1403.2382 [hep-ph]]; F. R. Joaquim and J. T. Penedo, Phys. Rev. D 90, no. 3, 033011 (2014) [arXiv:1403.4925 [hep-ph]]; M. Baumgart, D. Stolarski and T. Zorawski, Phys. Rev. D 90, no. 5, 055001 (2014) [arXiv:1403.6118 [hep-ph]]; H. Okada and K. Yagyu, Phys. Rev. D 90, no. 3, 035019 (2014) [arXiv:1405.2368 [hep-ph]]; W. G. Hollik, Phys. Rev. D 91, no. 3, 033001 (2015) [arXiv:1412.4585 [hep-ph]].

[6] W. Altmannshofer, C. Frugiuele and R. Harnik, JHEP 1412, 180 (2014) [arXiv:1409.2522 [hep-ph]].

[7] A. Bevan et al., arXiv:1411.7233 [hep-ph].

[8] W. Altmannshofer, R. Harnik and J. Zupan, JHEP 1311, 202 (2013) [arXiv:1308.3653 [hep-ph]].

[9] T. Moroi and M. Nagai, Phys. Lett. B 723, 107 (2013) [arXiv:1303.0668 [hep-ph]]; D. McKeen, M. Pospelov and A. Ritz, Phys. Rev. D 87, no. 11, 113002 (2013) [arXiv:1303.1172 [hep-ph]]; K. Fuyuto, J. Hisano, N. Nagata and K. Tsumura, JHEP 1312, 010 (2013) [arXiv:1308.6493 [hep-ph]]; T. Ibrahim, A. Itani and P. Nath, Phys. Rev. D 90, no. 5, 055006 (2014) [arXiv:1406.0083 [hep-ph]].

[10] J. P. Lees et al. [BaBar Collaboration], Phys. Rev. Lett. 109, 101802 (2012) [arXiv:1205.5442 [hep-ex]].

[11] M. Huschle et al. [Belle Collaboration], arXiv:1507.03233 [hep-ex]; Thomas Kuhr, these proceedings.

[12] R. Aaij et al. [LHCb Collaboration], arXiv:1506.08614 [hep-ex]; Greg Ciezarek, these proceedings.

[13] S. Fajfer, J. F. Kamenik and I. Nisandzic, Phys. Rev. D 85, 094025 (2012) [arXiv:1203.2654 [hep-ph]]; J. A. Bailey et al., Phys. Rev. Lett. 109, 071802 (2012) [arXiv:1206.4992 [hep-ph]].

[14] R. Aaij et al. [LHCb Collaboration], arXiv:1506.08777 [hep-ex]; Christian Linn, these proceedings.

[15] W. Altmannshofer and D. M. Straub, arXiv:1411.3161 [hep-ph]; arXiv:1503.06199 [hep-ph].

[16] A. Bharucha, D. M. Straub and R. Zwicky, arXiv:1503.05534 [hep-ph].

[17] R. Aaij et al. [LHCb Collaboration], Phys. Rev. Lett. 111, 191801 (2013) [arXiv:1308.1707 [hep-ex]]; LHCb Collaboration, LHCb-CONF-2015-002.

[18] S. Descotes-Genon, L. Hofer, J. Matias and J. Virto, JHEP 1412, 125 (2014) [arXiv:1407.8526 [hep-ph]]. 
[19] T. Hurth, F. Mahmoudi and S. Neshatpour, JHEP 1412, 053 (2014) [arXiv:1410.4545 [hep-ph]].

[20] S. Jäger and J. Martin Camalich, arXiv:1412.3183 [hep-ph].

[21] R. Aaij et al. [LHCb Collaboration], Phys. Rev. Lett. 113, 151601 (2014) [arXiv:1406.6482 [hep-ex]].

[22] B. Bhattacharya, A. Datta, D. London and S. Shivashankara, Phys. Lett. B 742, 370 (2015) [arXiv:1412.7164 [hep-ph]]; A. Greljo, G. Isidori and D. Marzocca, arXiv:1506.01705 [hep-ph]; L. Calibbi, A. Crivellin and T. Ota, arXiv:1506.02661 [hep-ph].

[23] S. Jäger and J. Martin Camalich, JHEP 1305, 043 (2013) [arXiv:1212.2263 [hep-ph]].

[24] A. Khodjamirian, T. Mannel, A. A. Pivovarov and Y.-M. Wang, JHEP 1009, 089 (2010) [arXiv:1006.4945 [hep-ph]]; A. Khodjamirian, T. Mannel and Y. M. Wang, JHEP 1302, 010 (2013) [arXiv:1211.0234 [hep-ph]].

[25] C. Bobeth, G. Hiller and G. Piranishvili, JHEP 0712, 040 (2007) [arXiv:0709.4174 [hep-ph]].

[26] S. Descotes-Genon, J. Matias and J. Virto, Phys. Rev. D 88, 074002 (2013) [arXiv:1307.5683 [hep-ph]]. W. Altmannshofer and D. M. Straub, Eur. Phys. J. C 73, 2646 (2013) [arXiv:1308.1501 [hep-ph]]. F. Beaujean, C. Bobeth and D. van Dyk, Eur. Phys. J. C 74, 2897 (2014) [Eur. Phys. J. C 74, 3179 (2014)] [arXiv:1310.2478 [hep-ph]]; T. Hurth and F. Mahmoudi, JHEP 1404, 097 (2014) [arXiv:1312.5267 [hep-ph]].

[27] D. Ghosh, M. Nardecchia and S. A. Renner, JHEP 1412, 131 (2014) [arXiv:1408.4097 [hep-ph]].

[28] G. Hiller and M. Schmaltz, Phys. Rev. D 90, 054014 (2014) [arXiv:1408.1627 [hep-ph]]; A. J. Buras, J. Girrbach-Noe, C. Niehoff and D. M. Straub, JHEP 1502, 184 (2015) [arXiv:1409.4557 [hep-ph]]; B. Gripaios, M. Nardecchia and S. A. Renner, JHEP 1505, 006 (2015) [arXiv:1412.1791 [hep-ph]]; I. de Medeiros Varzielas and G. Hiller, JHEP 1506, 072 (2015) [arXiv:1503.01084 [hep-ph]]; D. Becirevic, S. Fajfer and N. Kosnik, Phys. Rev. D 92, no. 1, 014016 (2015) [arXiv:1503.09024 [hep-ph]]; R. Alonso, B. Grinstein and J. M. Camalich, arXiv:1505.05164 [hep-ph].

[29] W. Altmannshofer, S. Gori, M. Pospelov and I. Yavin, Phys. Rev. D 89, 095033 (2014) [arXiv:1403.1269 [hep-ph]].

[30] A. Crivellin, G. D’Ambrosio and J. Heeck, Phys. Rev. Lett. 114, 151801 (2015) [arXiv:1501.00993 [hep-ph]]; A. Crivellin, G. D’Ambrosio and J. Heeck, Phys. Rev. D 91, no. 7, 075006 (2015) [arXiv:1503.03477 [hep-ph]]; C. Niehoff, P. Stangl and D. M. Straub, Phys. Lett. B 747, 182 (2015) [arXiv:1503.03865 [hep-ph]]; D. Aristizabal Sierra, F. Staub and A. Vicente, Phys. Rev. D 92, no. 1, 015001 (2015) [arXiv:1503.06077 [hep-ph]]; S. M. Boucenna, J. W. F. Valle and A. Vicente, arXiv:1503.07099 [hep-ph]; A. Crivellin, L. Hofer, J. Matias, U. Nierste, S. Pokorski and J. Rosiek, arXiv:1504.07928 [hep-ph]; A. Celis, J. Fuentes-Martin, M. Jung and H. Serodio, Phys. Rev. D 92, no. 1, 015007 (2015) [arXiv:1505.03079 [hep-ph]].

[31] W. Altmannshofer, S. Gori, M. Pospelov and I. Yavin, Phys. Rev. Lett. 113, 091801 (2014) [arXiv:1406.2332 [hep-ph]].

[32] G. Hiller and M. Schmaltz, JHEP 1502, 055 (2015) [arXiv:1411.4773 [hep-ph]].

[33] S. L. Glashow, D. Guadagnoli and K. Lane, Phys. Rev. Lett. 114, 091801 (2015) [arXiv:1411.0565 [hep-ph]]. 